\title{
Singularity Loci Analysis of the Position and Pose Adjustment Mechanism \\ Used for In-pipe Robot
}

\author{
Han $\mathrm{Ni}^{1, \mathrm{a}^{*}}$, Yulian Chang, Sheng Gao \\ ${ }^{1}$ Northeast Petroleum University, Daqing, 163318 China \\ ahljdqnh@163.com
}

Keywords: parallel mechanism, singularity loci

Abstract. A four-degree of freedom (4DOF) spatial parallel mechanism used as position and pose adjustment mechanism for manipulator is designed. By mechanism analysis, the conclusion that the force-influence coefficient matrix of the adjustment mechanism is the transposition of the velocity influence coefficient matrix is made and the force-influence coefficient matrix and nondimensional jacobian matrix in the initial state of mechanism are deduced. According to the judgment conditions of mechanism singularity loci, analyzing the singularity loci of the connection mechanism, author obtains the judgment criterion for in-pipe robot position and pose adjustment mechanism, laying the foundation for optimization design of mechanism parameters and motion trajectory planning.

When walking or working in the pipeline, the in-pipe robot needs to change the position and post in order to complete the movements of the angle, the axis rotation and the axial feed. At present, this type of robot is drived mostly based on the serial mechanism which is very difficult to realize these requirements, rather than on the parallel mechanism which can control the robot to change its position and pose. The four-degree of freedom spatial parallel mechanism used as position and pose adjustment mechanism is designed in order to adjust controllable the position and pose of the in-pipe robot, shown as Fig.1. The mechanism is comprised of moving platform, static platform, and three driving rod and a central rod which connect the platforms. The parallel mechanism have three rotation and a axial movements freedoms by coordinated control of four rods which expand and contract respectively.

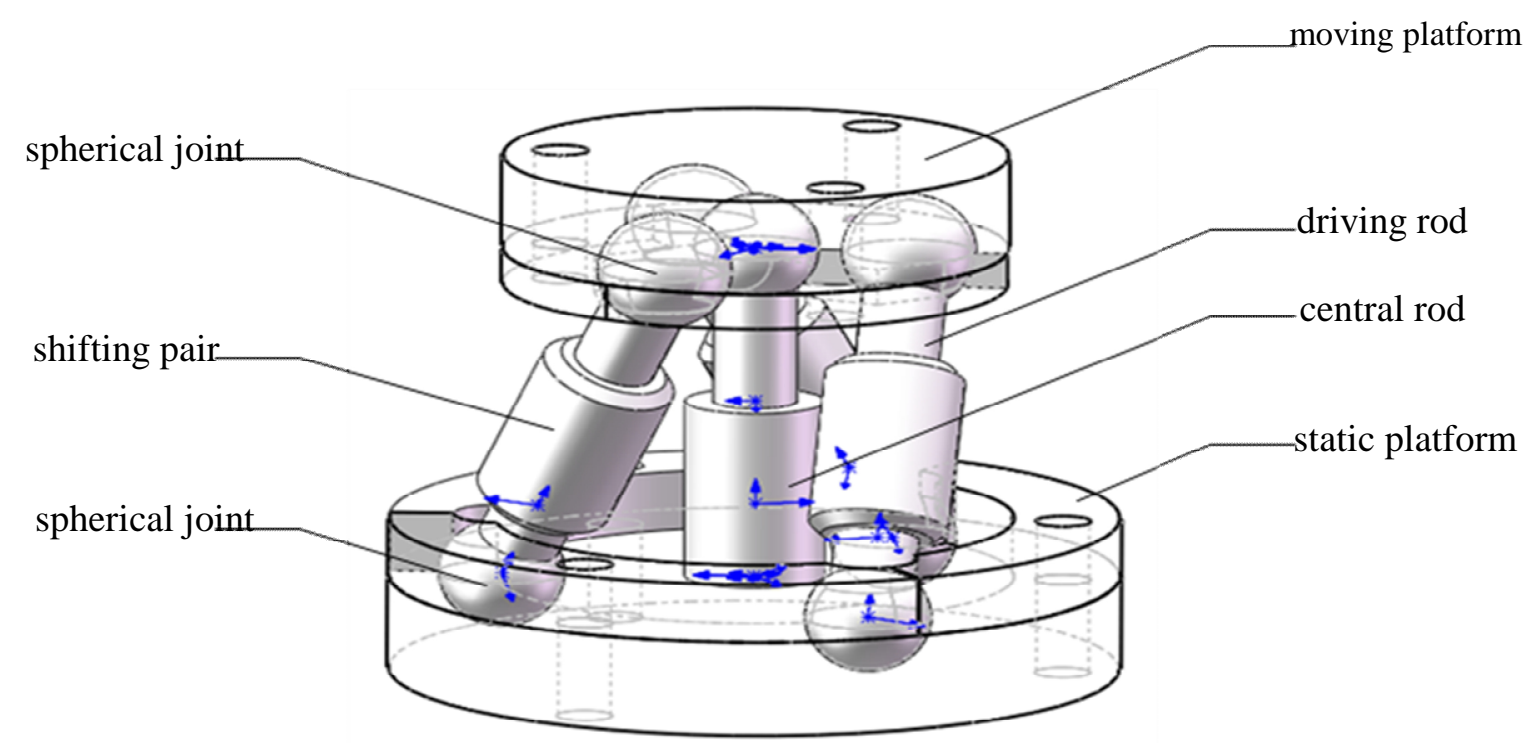

Fig. 1 the mold of the position and pose adjustment mechanism used for in-pipe robot

The optimization design of the parameters and the motion trajectory planning of the position and pose adjustment mechanism need to focus on the singularity loci of the mechanism in ordoer to avoid mechanism being out of control insult from the non steady state[1]. Domestic and foreign scholars 
research the singularity loci of the spatial parallel mechanism by using the screw theory[2,3], Jacobian matrix analysis[4], and Grassmann line geometry method[5] etc.. Here the singularity loci of the adjustment mechanism is researched by resolving the force Jacobian matrix, using the transpose relationship between the force Jacobian matrix and the velocity Jacobian matrix, and combining geometry analysis of the spatial mechanism.

\section{mechanism analysis}

The coordinates $P-x y z$ and $Q-X Y Z$ which fixedly connect the moving and static platform are established with circumcircle center of three hinged joint on the moving and static platform coordinates origin, shown as Fig.2. Three driving rods respectively have shifting pair in the middle, connect with platform by spherical joint on both ends, with a length $l$, and are called SPS driving rod. The three hinge joints on the platform are evenly distributed on the circumference with the center of the platform as the origin and with the distance $r$ between the hinge joint and origin as the radius of the circle. On end of the central $\operatorname{rod} \mathrm{A}_{4} \mathrm{~B}_{4}$ is vertically fixedly connected with $\mathrm{Q}$, the other end is connected with $\mathrm{P}$ by spherical joint. Both ends are connected together through shifting pair. The length of the central rod is $h$.

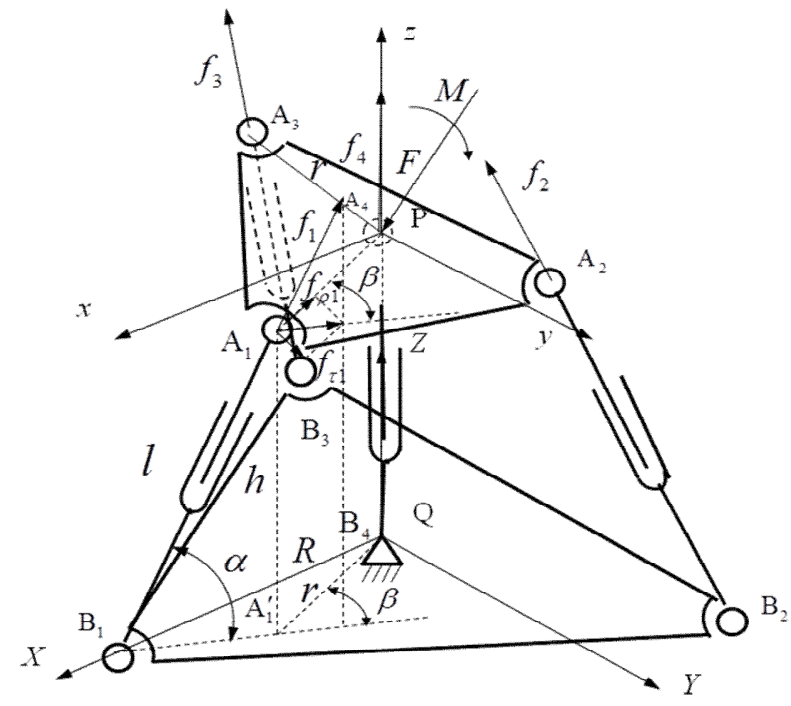

Fig.2 diagram of force on the position and post adjustment mechanism

The velocity of the center of the platform $P$ relative to the coordinates is set to $v$, the angular rate to $\boldsymbol{\omega}$, the movement speed of the driving rod to $\boldsymbol{v}_{L i}$, direction vector to $\boldsymbol{l}_{i}$. Then the velocity $\left(\boldsymbol{v}_{A i}\right)$ of the upper hinge joint can be expressed as:

$$
\boldsymbol{v}_{A i}=\boldsymbol{v}+\boldsymbol{\omega} \times \boldsymbol{r}_{i} \quad i=1,2,3,4
$$

The expand and contract speed ( $\left.\boldsymbol{v}_{L i}\right)$ of each driving rod is the projection of $\boldsymbol{v}_{A i}$ in the direction of $\boldsymbol{l}_{i}$, so:

$$
\begin{gathered}
\boldsymbol{v}_{L i}=\boldsymbol{v}_{A i} \boldsymbol{g}_{i}=\left(\boldsymbol{v}+\boldsymbol{\omega} \times \boldsymbol{r}_{i}\right) \boldsymbol{g}_{i}=\boldsymbol{l}_{i} \boldsymbol{g}+\left(\boldsymbol{r}_{i} \times \boldsymbol{l}_{i}\right) \boldsymbol{g} \boldsymbol{v}=\boldsymbol{J} \boldsymbol{V} \quad i=1,2,3,4 \\
\boldsymbol{V}=\boldsymbol{J}^{-1} \boldsymbol{v}_{L i} \quad i=1,2,3,4
\end{gathered}
$$

The external force of the center of moving platform is set to $\boldsymbol{F}=\left[\begin{array}{lll}F_{x} & F_{y} & F_{z}\end{array}\right]$ and the torque to $\boldsymbol{M}=\left[\begin{array}{lll}M_{x} & M_{y} & M_{z}\end{array}\right]$, according to the principle of virtual work, then: 


$$
\begin{gathered}
\boldsymbol{F}_{l}^{\mathrm{T}} \boldsymbol{v}_{L i}+\left[\begin{array}{c}
\boldsymbol{F} \\
\boldsymbol{M}
\end{array}\right]^{\mathrm{T}} \boldsymbol{V}=0 \quad i=1,2,3,4 \\
{\left[\begin{array}{c}
\boldsymbol{F} \\
\boldsymbol{M}
\end{array}\right]=-\boldsymbol{J}^{\mathrm{T}} \boldsymbol{F}_{l} \quad i=1,2,3,4}
\end{gathered}
$$

Equation (5) is the input/output equation for mechanism force, wherein $\boldsymbol{J}^{\mathrm{T}}$ is the influence coefficient matrix of the position and pose adjustment mechanism of the robot. $J$ is the velocity influence coefficient matrix. Analysis of equation (3) and equation (5) shows that force influence coefficient matrix and velocity influence coefficient matrix are transpose relationship mutually.

\section{solving the force Jacobian matrix}

The driving force of the driving rod is set to $f_{i}$. Point $\mathrm{P}$ is constrained by the central $\operatorname{rod} \mathrm{A}_{4} \mathrm{~B}_{4}$. Total force in the direction of $\mathrm{X}, \mathrm{Y}$ axes is zero. The moving platform and static platform are parallel to each other. The angle between the driving rod and the static platform is $\alpha$ The driving rod is subjected to tension or pressure, because there does not produce the torque at the hinge joint $\mathrm{S}$. The moving platform rotates a certain angle around the $\mathrm{Z}$ axis under the action of the driving rod. The projection of the driving force $f_{i}(\mathrm{i}=1,2,3)$ on the moving platform deflects from overlapping the line $\mathrm{A}_{i} \mathrm{P}$ to having the angle $\beta$ with the line $\mathrm{A}_{i} \mathrm{P}$.

The static equilibrium equation shows that the axial force $f_{z i}$, normal force $f_{\rho i}$ and tangential force $f_{\tau i}$ that the moving platform subjects at point $\mathrm{A}_{i}$ are respectively:

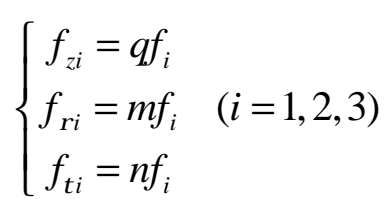

Wherein, $\mathrm{q}, \mathrm{m}, \mathrm{n}$ are intermediate variables, $q=\sin \alpha, m=\cos \alpha \cos \beta, n=\cos \alpha \sin \beta$

When the moving platform is in equilibrium, the forces of each driving rod and the load (force and torque) that the moving platform is subjected are balanced. $f_{5}, f_{6}$ are the restraint reaction force. According to the characteristics of sufferance force, there are:

$$
\left\{\begin{array}{l}
F_{x}=-m f_{1}+\left(\frac{1}{2} m-\frac{\sqrt{3}}{2} n\right) f_{2}+\left(\frac{1}{2} m+\frac{\sqrt{3}}{2} n\right) f_{3}-f_{5} \\
F_{y}=n f_{1}+\left(-\frac{1}{2} n-\frac{\sqrt{3}}{2} m\right) f_{2}+\left(-\frac{1}{2} n+\frac{\sqrt{3}}{2} m\right) f_{3}-f_{6} \\
F_{z}=q f_{1}+q f_{2}+q f_{3}+f_{4} \\
M_{x}=\frac{\sqrt{3}}{2} r q f_{2}-\frac{\sqrt{3}}{2} r q f_{3} \\
M_{y}=-r q f_{1}+\frac{1}{2} r q f_{2}+\frac{1}{2} r q f_{3} \\
M_{z}=r n f_{1}+r n f_{2}+r n f_{3}
\end{array}\right.
$$

In the matrix form: 


$$
\left[\begin{array}{llllll}
F_{x} & F_{y} & F_{z} & M_{x} & M_{y} & M_{z}
\end{array}\right]^{\mathrm{T}}=\boldsymbol{J}^{\mathrm{T}}\left[\begin{array}{llllll}
f_{1} & f_{2} & f_{3} & f_{4} & f_{5} & f_{6}
\end{array}\right]^{\mathrm{T}}
$$

The elements relating to rotation of the Jacobian matrix are divided by the characteristic length $a_{0}$, and then the new dimensionless Jacobian matrix is obtained. Non dimensional force Jacobian matrix $\boldsymbol{J}_{w}^{\mathrm{T}}$ is denoted as:

$$
\boldsymbol{J}_{w}^{\mathrm{T}}=\boldsymbol{H} \boldsymbol{J}^{\mathrm{T}}
$$

Wherein, $\boldsymbol{H}=\left[\begin{array}{cc}\boldsymbol{E}_{3 \times 3} & 0 \\ 0 & \boldsymbol{A}_{0}\end{array}\right], \quad \boldsymbol{E}_{3 \times 3}$ is 3 order unit matrix, $\boldsymbol{A}_{0}=\operatorname{diag}\left(\frac{1}{a_{0}}, \frac{1}{a_{0}}, \frac{1}{a_{0}}\right)$ 。

Then dimensionless velocity Jacobian matrix $\boldsymbol{J}_{w}$ :

$$
\boldsymbol{J}_{w}=\left(\boldsymbol{J}_{w}^{\mathrm{T}}\right)^{\mathrm{T}}=\left[\begin{array}{cccccc}
-m & n & q & 0 & \frac{-r q}{a_{0}} & \frac{r n}{a_{0}} \\
\frac{1}{2} m-\frac{\sqrt{3}}{2} n & -\frac{1}{2} n-\frac{\sqrt{3}}{2} m & q & \frac{\sqrt{3}}{2 a_{0}} r q & \frac{1}{2 a_{0}} r q & \frac{r n}{a_{0}} \\
\frac{1}{2} m+\frac{\sqrt{3}}{2} n & -\frac{1}{2} n+\frac{\sqrt{3}}{2} m & q & -\frac{\sqrt{3}}{2 a_{0}} r q & \frac{1}{2 a_{0}} r q & \frac{r n}{a_{0}} \\
0 & 0 & 1 & 0 & 0 & 0 \\
-1 & 0 & 0 & 0 & 0 & 0 \\
0 & 1 & 0 & 0 & 0 & 0
\end{array}\right]
$$

\section{judgment criterion for position and pose}

According to the research of Huang, whether the parallel mechanism is in the singularity loci or not can be judged by judging if the Jacobian matrix equals to zero.

(i) When $\alpha=0$ or $\pi, q=0$. Here, the angle between three SPS driving rod and static platform is zero, the height of the joint $h=0$. As $r+l>R$, and $h \neq 0$, it is impossible that moving platform moves to attach to the static platform, this extreme position is not exist.

(ii) When $\cos \alpha=0$, that is $\alpha=\pi / 2, n=0$, if $R=r$, three SPS driving rods are perpendicular to the static platform. If $R>r$, in the course of the movement of the moving platform, three SPS driving rod can not perpendicular to the static platform at the same time.

(iii) When $\sin \beta=0, n=0$. Here moving platform parallel to the static platform, three points where the platform connects three SPS driving rods form an equilateral triangle. The extend lines of the projections of the movement speed of the driving rod or driving force on the platform intersect at the center of the equilateral triangle. Such the singularity loci of the mechanism is constructed. The moving platform can not withstand any load.

(iv) Conclusion

A four-degree of freedom spatial parallel mechanism used as connection mechanism of the robot is designed to meet the requirements of the position and pose of the in-pipe robot adjustment under the work condition. The moving platform of the position and pose adjustment mechanism can rotate along the $\mathrm{X}, \mathrm{Y}$ and $\mathrm{Z}$ axis and move along the $\mathrm{Z}$ axis, which can meet the requirements of position and pose adjustment, such as movement of the angle, the axis rotation and the axial feed. 
By mechanism analysis, the conclusion that the force Jacobian matrix of the mechanism is the transposition of the velocity Jacobian matrix is made and the force-influence coefficient matrix and nondimensional jacobian matrix in the initial state of mechanism are deduced. According to the judgment conditions of mechanism singularity loci, author analyze the singularity loci of the position and pose adjustment mechanism, lay the foundation for optimization design of mechanism parameters and motion trajectory planning.

\section{References}

[1]Nguyeg C.C.Analysis and implementation of a 6-DOF Stewart-platform-based robotic wrist[J]. Computers and Electrical Engineering, 1997, 17(3): 191-204.

[2] Kong X.W, Jaime G.A, Rico J.M. Kinematic and singularity analysis of a 4-DOF parallel manipulator using screw theory [J]. Mechanism and Machine Theory, 2006, 41(9):1048-1061.

[3] Zlatanov D, Bonev I.A, Gosselin C.M. Constraint singularities of parallel mechanisms [C].Proceedings of the 2002 IEEE International Conference on Robotics. Washington, DC, 2002:496-505.

[4] Fichter E.F. A Stewart platform-based manipulator [J]. General Theory and Practical Construction. International Journal of Robotic Research, 1986, 5(2): 157-182.

[5] Wolf A, Qttaviano E, Shoham M, Ceccarelli M. Application of lone geometry and linear complex approximation to singularity analysis of the 3-DOF Ca Pa Man parallel manipulator[J]. Mechanism and Machine Theory, 2004, 39(1): 75-95. 